\title{
HERMITE INTERPOLATION AND $p$-ADIC EXPONENTIAL POLYNOMIALS
}

\author{
Dedicated to George Szekeres on his 65th birthday
}

A. J. van der POORTEN

(Received 26 March, 1975)

Communicated by Jennifer Seberry Wallis

\begin{abstract}
By employing a precise form of the Hermite interpolation formula we obtain a best possible bound for the number of zeros of $p$-adic exponential polynomials. As companion to this quantitative result we give a best possible bound on the coefficients, if the exponential polynomial is small at sufficiently many points.
\end{abstract}

\section{Introduction}

Our purpose in this paper is to state and prove some auxiliary results in the theory of $p$-adic transcendental numbers. In so doing we have endeavoured to avoid paraphrasing the complex techniques and have used methods which may be inappropriate or inefficient in the complex case. We profit by obtaining best possible results for the number of zeros, and, respectively, small points of $p$-adic exponential polynomials. We also obtain an extrapolation lemma which we claim to be more efficient than is extrapolation with the aid of the Schnirelman integral.

Specifically, let

$$
F(z)=\sum_{k=1}^{m} \sum_{s=1}^{p(k)} \frac{b_{k s}}{(s-1) !} z^{s-1} e^{\omega_{k} z}
$$

be a $p$-adic exponential polynomial of degree $\sigma=\Sigma \rho(k)$, whose frequencies $\omega_{1}$ satisfy $\left|\omega_{k}\right|_{p} \leqq p^{-(1 /(p-1)+\theta)}$, some $\theta>0$, and $\min _{h \neq k}\left|\omega_{k}-\omega_{n}\right|_{p} \geqq p^{-\delta_{k}}$. Then the number of zeros of $F$ in the disc $\left\{z \in \Omega_{p}:|z|_{p} \leqq 1\right\}$ is less than

$$
N=(\sigma-1)+\max _{0 \leqq r<p-1}\left\{\left[\log _{p}(\sigma+r)\right]-r /(p-1)\right\} / \theta,
$$


or $F$ vanishes identically. If, further, $\alpha_{1}, \cdots, \alpha_{n}$ are distinct points of $\Omega_{p}$, satisfying $\left|\alpha_{h}\right|_{p} \leqq 1$ and $\min _{k \neq h}\left|\alpha_{h}-\alpha_{k}\right|_{p} \geqq p^{-e}$ and $\tau(1), \cdots, \tau(n)$ are positive integers with sum $\Sigma \tau(h)=T$, where $T \geqq N$, then $(\sigma>1$ and)

$$
\max _{\substack{i \leq n \leq n \\ t \leq t \leq \tau(h)}}\left|F^{(t-1)}\left(\alpha_{h}\right) /(t-1) !\right|_{p} \leqq p^{-x}
$$

implies that, for all pairs $(k, s)$,

$$
\left|b_{k s}\right|_{p}<p^{-x} p^{(T-1) e} p^{(\sigma-s) \delta_{k}} .
$$

I am indebted to John Coates who alerted me to the Hermite interpolation formula as it appears in Schinzel (1967), and to the Department of Pure Mathematics and Mathematical Statistics of the University of Cambridge, where I wrote this paper whilst on sabbatical leave from the University of New South Wales.

\section{The interpolation formula}

THEOREM 1. Let $n$ be a positive integer and $\tau(1), \cdots, \tau(n)$ non-negative integers with sum $\Sigma_{\tau}(h)=T$. Let $F(z)$ be a function sufficiently many times differentiable at each of the $n$ distinct points $\alpha_{1}, \cdots, \alpha_{n}$.

Then the unique polynomial $P(z)$ of degree $\leqq T-1$ with the property that

$$
P^{(t-1)}\left(\alpha_{h}\right)=F^{(t-1)}\left(\alpha_{h}\right), \quad \text { for } h=1, \cdots, n ; t=1, \cdots, \tau(h)
$$

is given by the formula

$$
\begin{aligned}
& P(z)=\sum_{h=1}^{n} \sum_{i=1}^{r(h)} F^{(t-1)}\left(\alpha_{h}\right)\left\{\prod_{\substack{k=1 \\
k \neq h}}^{n}\left(\frac{z-\alpha_{k}}{\alpha_{h}-\alpha_{k}}\right)^{\tau(k)} \cdot \frac{\left(z-\alpha_{h}\right)^{t-1}}{(t-1) !} \cdot\right. \\
& \sum_{\lambda} \prod_{r=t+1}^{\tau(h)} \frac{\left(\frac{\partial}{\partial \alpha_{h}}\right)^{\lambda(r)}\left\{\left(z-\alpha_{h}\right) \prod_{\substack{j=1 \\
i \neq h}}^{n}\left(\alpha_{h}-\alpha_{i}\right)^{r(j)}\right\}(-1)^{\max (0, \lambda(r)-1)}}{\lambda(r) ! \prod_{\substack{j=1 \\
j \neq h}}^{n}\left(\alpha_{h}-\alpha_{i}\right)^{\tau(j)}}
\end{aligned}
$$

where the sum is over all non-negative integer sets $\lambda(t+1), \cdots, \lambda(\tau(h))$ with sum $\tau(h)-t$ and such that $\lambda(r) \leqq r-t$ and such that $\lambda(\tau(h)) \geqq 1$ (if $t=\tau(h)$ the sum is of course empty.)

Proof. Since the ranges of the various indices will mostly be quite clear we avoid their explicit mention. Our proof is based on the observation that we are obtaining the confluent case of the well-known Lagrange interpolation 
formula. Accordingly we introduce $T$ parameters $\alpha_{k s}$ indexed by the pairs $(k, s), k=1, \cdots, n ; s=1, \cdots, \tau(k)$. We suppose these parameters to be distinct. When, below we speak of taking the limit, or use the symbol lim, we imply that we will allow confluence to take place, that is $\alpha_{k s} \rightarrow \alpha_{k}$ for all the pairs $(k, s)$.

Denote by $D$ the $T \times T$ determinant with typical entry $\alpha_{k s}^{\lambda}$; here columns are indexed by the pairs $(k, s)$ lexicographically ordered, and the rows by $\lambda$, $\lambda=1, \cdots, T$. Denote the cofactor of $\alpha_{k s}^{\lambda-1}$ by $D_{k s, \lambda}$.

It is now simply the Lagrange interpolation formula to observe that the unique polynomial $Q(z)$ of degree $T-1$ with the property that

$$
Q\left(\alpha_{h t}\right)=F\left(\alpha_{h t}\right) \quad \text { for all }(h, t)
$$

is given by the formula

$$
Q(z)=\sum_{h} \sum_{t} F\left(\alpha_{h t}\right) \sum_{\lambda} \frac{D_{h t, \lambda} z^{\lambda-1}}{D}
$$

We will obtain the formula for the interpolation polynomial $P(z)$ by taking the limit in the formula for $Q(z)$.

Accordingly denote by $\Delta$ the $T \times T$ determinant with typical entry $((\lambda-1) ! /(\lambda-s) !) \alpha_{k}^{\lambda-s}$; here columns and rows are indexed as above in $D$. Denote the cofactor of the typical entry by $\Delta_{k s . \lambda}$.

Then by what is no more than a repeated application of the so-called l'Hôpital's rule we obtain from (2) the formula

$$
P(z)=\sum_{h} \sum_{t} F^{(t-1)}\left(\alpha_{h}\right) \sum_{\lambda} \frac{\Delta_{h t, \lambda} z^{\lambda-1}}{\Delta} .
$$

Actually it is quite straightforward to check that $P(z)$ as defined by (3) has the $T$ required properties and is a polynomial of degree $\leqq T-1$. Since these conditions clearly define $P(z)$ uniquely the truth of the formula (3) follows. As the formula of the theorem is simply an explicit reformulation of (3) we need only find an explicit expression for the sum

$$
\sum_{\lambda} \frac{\Delta_{h l \lambda} z^{\lambda-1}}{\Delta}
$$

which we achieve by writing it as the following limit

$$
\sum_{\lambda} \frac{\Delta_{h l, \lambda} z^{\lambda-1}}{\Delta}=\lim \frac{\left\{\prod_{(k, s) \neq(h, t)}\left(\frac{\partial}{\partial \alpha_{k s}}\right)^{s-1}\right\}\left(D \cdot \prod_{(k, s) \neq(h, t)}\left(\frac{z-\alpha_{k s}}{\alpha_{h t}-\alpha_{k s}}\right)\right)}{\Delta} .
$$

We remark that the determinant $\Delta$ is explicitly given by 


$$
\Delta=\prod_{(h, t)}\left\{(t-1) ! \prod_{k=1}^{n-1}\left(\alpha_{h}-\alpha_{k}\right)^{\tau(k)}\right\}
$$

(For details see van der Poorten (1975), see also van der Poorten (1970), particularly p. 34-35) The formula of the theorem now follows from (4) after a painful struggle which it seems pointless to detail. The principal point to notice is that the derivations in (4) are mostly 'used up' in eliminating from $D$ those factors which would vanish on taking the limit.

Remarks. (i) Although the idea of the formula (1) dates back at least to Hermite, I do not know of it having been stated before in so explicit a shape. In any event this explicit form does not appear to have been used, as such, in applications. Mahler's identities in Mahler (1932), and again Mahler (1967b), get very close, and I have a rather inadequate application of what is essentially the same formula in van der Poorten (1970). My recognition of the identity (3) and thence the interpolation formula derives from Mahler's ideas, but when in van der Poorten (1970) I used the idea of confluence explicitly, I had not earlier met it in this kind of context.

(ii) The unpleasant sum over $\lambda$ in the interpolation formula is of no matter in estimating in the non-archimedean case when we need only consider the 'worst' term in the sum. In this case one obtains particularly efficient estimates. However even in the archimedean case one can obtain useful estimates as is evidenced by Mahler (1967b).

(iii) We have retained maximum detail in the interpolation formula since doing so presents no extra difficulties and even assists in revealing pattern.

\section{Preliminaries}

Let $p$ be a rational prime, henceforth fixed, and denote by $\Omega_{p}$ the algebraic closure of the $p$-adic completion $Q_{p}$ of the field of rational numbers. We denote the valuation of $\Omega_{p}$ by ||$_{p}$ so normalised that $|p|_{p}=p^{-1}$, and for $\alpha \in \Omega_{p}$ we write $\operatorname{ord}_{p} \alpha=-\log _{p}|\alpha|_{p}=-\log |\alpha|_{p} / \log p$. In the sequel we shall mostly suppress the suffix $p$.

We commence by giving estimates for values of the interpolation polynomial in some special cases. The notation is that of the theorem, $\alpha_{1}, \cdots, \alpha_{n} \in \Omega_{p}$ and the function $F$ and its derivatives take values in $\Omega_{p}$.

Lemma 1. Suppose ord $\alpha_{h} \geqq \theta>0$ and $\max _{k \neq h}$ ord $\left(\alpha_{h}-\alpha_{k}\right) \leqq \varepsilon+\theta$ for $h, k=1, \cdots, n$. Then for $\lambda=1,2, \cdots, T$ the formula (1) implies ord $P^{(\lambda-1)}(0) \geqq$ $\min _{(h, t)}\left\{\right.$ ord $\left.F^{(t-1)}\left(\alpha_{h}\right)-(T-t) \varepsilon-(\lambda-t) \theta-\operatorname{ord}(t-1) !+\operatorname{ord}(\lambda-1) !\right\}$

Proof. The assertion is essentially immediate from (1). One notices that 
necessarily $\varepsilon \geqq 0$ so that in the 'bad' term we must suppose that we lose, in differentiating with respect to $\alpha_{h}$, as much as $(\tau(h)-t)(\varepsilon+\theta)$. Differentiating with respect to $z$ costs no more than $(\lambda-1) \theta$ and gains ord $(\lambda-1)$ !

Lemma 2. Write $n=R$ and let $\alpha_{1}=1, \cdots, \alpha_{R}=R$. Further write $\tau(1)=$ $\cdots=\tau(n)=S$, and let $l$ be an integer greater than $R$. Then the formula (1) implies

$$
\begin{aligned}
\text { ord } P(l) & \geqq \min _{(h, t)}\left\{\operatorname{ord} F^{(t-1)}(h)+S \text { ord }\left(\begin{array}{c}
l-1 \\
R
\end{array}\right)+S \operatorname{ord}\left(\begin{array}{l}
R-1 \\
h-1
\end{array}\right)\right. \\
& +S \operatorname{ord} R+(t-1) \operatorname{ord}(l-h)-S \operatorname{ord}(l-h)-\operatorname{ord}(t-1) ! \\
& -(S-t) \max \{[\log (R-h) / \log p],[\log (h-1) / \log p], \operatorname{ord}(l-h)\} .
\end{aligned}
$$

Proof. Substitute in (1) and strategically combine factors.

We will require the following concept due to Mahler (1935), 264-266:

Definition. A ( $p$-adic) power series $\Sigma_{i \geq 0} f_{i} z^{i}, f_{i} \in \Omega_{p}$ is said to be a normal series if $\left|f_{i}\right| \leqq 1$ for $i=0,1,2, \cdots$ and $\lim _{i \rightarrow \infty}\left|f_{i}\right|=0$. A ( $p$-adic) function $f(z)=\Sigma f_{i} z^{i}$ representable by a normal series is called a normal function.

Lemma 3 (Mahler). (i) A normal series converges to a (normal) function $f(z)$ for all $z \in \Omega_{p}$ such that $|z| \leqq 1$.

(ii) If $f(z)=\Sigma f_{i} z^{\prime}$ is a normal series then for each $z_{0} \in \Omega_{p}$ such that $\left|z_{0}\right| \leqq 1$ the series

$$
f(z)=\Sigma f_{i}\left(z_{0}\right)\left(z-z_{0}\right)^{i}, \text { where } f_{i}\left(z_{0}\right)=f^{(i)}\left(z_{0}\right) / i !=\sum_{j}\left(\begin{array}{l}
j \\
i
\end{array}\right) f_{i} z_{0}^{j-i}
$$

is again a normat series (accordingly the concept of 'normal function' is well-defined)

(iii) If $f$ is a normal function with zeros $\alpha_{1}, \cdots, \alpha_{n}$ of multiplicities $\tau(1), \cdots, \tau(n)$ respectively, where $\left|\alpha_{h}\right| \leqq 1$ for $h=1, \cdots, n$, then

$$
f(z)=\prod_{h}\left(z-\alpha_{h}\right)^{\tau(h)} g(z),
$$

where $g$ is a normal function.

Proof. (i) is obvious and (ii) checks easily on recalling that combinatorial coefficients are rational integers, whilst (iii) follows stepwise (that is, by induction on $n$ ) using (ii).

NOTE. If $F(z)$ is represented by a $p$-adic power series converging in some open neighbourhood of the origin, then there exist real numbers $l$ and $\theta$ such that $p^{\prime} F\left(p^{\theta} z\right)$ is a normal function of $z$. 
LemMa 4. The interpolation polynomial of Lemma 1 is a normal function provided that the quantity

$$
-l^{\prime}=\min _{(h . s)}\left\{\text { ord } F^{(t-1)}\left(\alpha_{h}\right)-(T-t) \varepsilon-\operatorname{ord}(t-1) !-(T-t) \theta\right\}
$$

is non-negative; in any event $p^{\prime} P(z)$ is a normal function.

Proof. By Lemma 1, ord $P^{(\lambda-1)}(0)-$ ord $(\lambda-1) !>-l^{\prime}$ for $\lambda=1, \cdots, T$ and $P$ has degree $\leqq T-1$.

These preliminaries allow us to state the principal interpolation lemma; the notation is that of the theorem, together with $\left|\alpha_{n}\right| \leqq 1, h=1, \cdots, n$.

Lemma 5. Suppose that $p^{\prime} F(z)$ and $p^{\prime \prime} P(z)$, where $P$ is the interpolation polynomial, are normal functions. Then for $z_{0} \in \Omega_{p},\left|z_{0}\right| \leqq 1$ and $\lambda=1,2, \cdots, T$ we have

ord $F^{(\lambda-1)}\left(z_{0}\right)$

$$
\geqq \min \left\{\operatorname{ord} P^{(\lambda-1)}\left(z_{0}\right),\left\{\operatorname{ord}\left(\frac{d}{d z}\right)^{\lambda-1} \prod_{h}\left(z \div \alpha_{h}\right)^{\tau(h)}\right\}_{z=z_{0}}-\max \left\{l, l^{\prime}\right\}\right\} .
$$

Proof. Write $l^{\prime \prime}=\max \left\{l, l^{\prime}\right\}$. Then both $p^{l^{\prime}} F(z)$ and $p^{l^{\prime \prime}} P(z)$ are normal functions so that by Lemma 3 we have

$$
p^{\prime \prime}(F(z)-P(z))=\prod\left(z-\alpha_{h}\right)^{r(h)} G(z),
$$

where $G(z)$ is a normal function. Hence

$$
\operatorname{ord}\left\{\left(\frac{d}{d z}\right)^{\wedge-1} \prod\left(z-\alpha_{h}\right)^{r(h)} G(z)\right\}_{z=z_{0}} \geqq \operatorname{ord}\left\{\left(\frac{d}{d z}\right)^{\lambda-1} \prod\left(z-\alpha_{h}\right)^{r(h)}\right\}_{z-z_{0}}
$$

because ord $G^{(\mu)}\left(z_{0}\right)-$ ord $\mu ! \geqq 0$ for all $\mu=0,1, \cdots$. On noticing that $F(z)=$ $P(z)+p^{-1 *} \Pi\left(z-\alpha_{h}\right)^{\tau(h)} G(z)$ the assertion follows.

Lemma 6. Let $\mu$ be a non-negative rational integer. Then

$$
\text { ord } \mu ! \leqq \mu /(p-1)-p^{-\left(\log _{p} \mu\right)} \mu /(p-1) .
$$

Proof. Ord $\mu !=\sum_{i=1}^{\infty}\left[\mu / p^{i}\right] \leqq \mu /(p-1)-p^{-1} \mu /(p-1)$, where $l$ is the rational integer such that $p^{\prime} \leqq \mu<p^{l+1}$.

\section{Exponential polynomials}

Definition. A ( $p$-adic) exponential polynomial is a function of the shape

$$
F(z)=\sum_{k=1}^{m} \sum_{s=1}^{\rho(k)} a_{k s} z^{s-1} e^{\omega_{k} z} \quad a_{k s} \in \Omega_{p}, \omega_{k} \in \Omega_{p}
$$

and ord $\omega_{k}>1 /(p-1), k=1, \cdots, m$. We refer to the $a_{k s}$ as the coefficients of $F$ 
and the $\omega_{k}$ as the frequencies of $F$, and write $\sigma=\Sigma \rho(k)$, which we call the degree of $F$. We assume the frequencies $\omega_{1}, \cdots, \omega_{m}$ to be distinct.

Lemмa 7. Let $F$ be an exponential polynomial as defined above, and suppose that its frequencies satisfy, for each $k, \max _{k \neq k} \operatorname{ord}\left(\omega_{k}-\omega_{k}\right) \leqq$ $\delta_{k}-\theta, k, h=1, \cdots, m$. Then the coefficients of $F$ satisfy

$$
\begin{aligned}
\text { ord } a_{k s}>\min _{1 \leqq \lambda \leqq \sigma}\left\{\operatorname{ord} F^{(\lambda-1)}(0)-\right. & (\sigma-s) \delta_{k}-\operatorname{ord}(s-1) ! \\
+ & (\sigma-\lambda) /(p-1)+(\sigma-s) \theta\}, \\
& \quad \text { for each } k=1, \cdots, m ; s=1, \cdots, \rho(k) .
\end{aligned}
$$

PRoof. If $\Delta$ denotes the $\sigma \times \sigma$ determinant with typical entry $((\lambda-1) ! /(\lambda-s) !) \omega_{k}^{\lambda-s}$ and $\Delta_{k s, \lambda}$ denotes the cofactor of the typical entry, then

$$
a_{k s}=\sum_{\lambda=1}^{\sigma} \frac{\Delta_{k s, \lambda}}{\Delta} F^{(\lambda-1)}(0)
$$

since this is just Cramer's rule. The estimate then follows as in the proofs of Theorem 1 and Lemma 1.

Lemma 8. Let $F$ be an exponential polynomial as defined above. Then either $F$ vanishes identically or given any real number $\nu>0$ there exists a real number 1 such that $p^{\prime} F(z)$ is a normal function but $p^{1-\nu} F(z)$ is not a normal function.

Proof. Firstly observe that if ord $\omega>1 /(p-1)$ then, for $i=0,1,2, \cdots$ ord $\omega^{i}-$ ord $i ! \geqq 0$ by Lemma 6 , and even $\lim _{i \rightarrow x}\left(\right.$ ord $\omega^{i}-$ ord $\left.i !\right)=\infty$, so $e^{\omega z}$ is a normal function. Hence there is a real number $l^{\prime}$, say so that the finitely many conditions ord $a_{k s}-l^{\prime} \geqq 0$ are satisfied, such that $p^{\prime} F(z)$ is a normal function. Now suppose that $F$ does not vanish identically, in which case, clearly, not all its coefficients $a_{k s}$ can vanish. It follows from (5) above, and the explicit evaluation of $\Delta$ towards the end of the proof of Theorem 1 which shows that $\Delta \neq 0$, that not all of the derivatives $F^{(\lambda-1)}(0), \lambda=1,2, \cdots, \sigma$, can vanish either. Accordingly there is a $\lambda$ such that there exists a real number $l^{\prime \prime}$ such that ord $F^{(\lambda-1)}(0)-\operatorname{ord}(\lambda-1) !+l^{\prime \prime}<0$. Then $p^{\prime \prime} F(z)$ is not a normal function, and it follows that the set $\left\{l^{\prime} \in \mathbf{R}: p^{\prime} F(z)\right.$ is a normal function $\}$ is bounded below. Since this set is clearly an interval of $\mathbf{R}$, and is non-trivial, this completes the proof of the lemma.

The following results are the $p$-adic analogues of results of Tijdeman (1973) and of Mahler (1967a) and Tijdeman (1971). We have avoided the detail of Tijdeman (1973) and Mahler (1967a) in favour of precision in another direction, but this detail could easily be reinserted. 
THEOREM 2. Let F be a p-adic exponential polynomial

$$
F(z)=\sum_{k=1}^{m} \sum_{s=1}^{\rho(k)} a_{k s} z^{s-1} e^{\omega_{k}{ }^{2}},
$$

with coefficients $a_{k s}$, not all zero, in $\Omega_{p}$, and distinct frequencies $\omega_{k}$ in $\Omega_{p}$ satisfying ord $\omega_{k} \geqq 1 /(p-1)+\theta$, some $\theta>0$, and $\max _{h \neq k}$ ord $\left(\omega_{k}-\omega_{h}\right) \leqq \delta_{k}$, $(k, h=1, \cdots, m)$. Let $\sigma=\sum \rho(k)$ be the degree of $F$.

Further, let $\alpha_{1}, \cdots, \alpha_{n}$ be distinct points of $\Omega_{p}$ satisfying ord $\alpha_{n} \geqq 0$ and $\max _{k \neq h}$ ord $\left(\alpha_{h}-\alpha_{k}\right) \leqq \varepsilon, \quad(h, k=1, \cdots, n)$, and let $\tau(1), \cdots, \tau(n)$ be nonnegative integers with sum $T=\Sigma \tau(h)$.

Then

$$
T \geqq(\sigma-1)+[\log (\sigma+p-1) / \log p] / \theta,
$$

and

$$
\operatorname{ord} F^{(t-1)}\left(\alpha_{h}\right) \geqq \eta \quad \text { for } h=1, \cdots, n ; t=1, \cdots, \tau(h),
$$

imply that

$$
\begin{aligned}
& \text { ord } \begin{aligned}
a_{k s}> & \eta-(T-1) \varepsilon-(\sigma-s) \delta_{k}-\operatorname{ord}(s-1) ! \\
& -[\log (\sigma+p-1) / \log p]+(\sigma-1) /(p-1) \\
& \quad+\min _{(h, t)}\{(t-1) \varepsilon-\operatorname{ord}(t-1) !\}
\end{aligned} \\
& \quad \text { for all } k=1, \cdots, m ; s=1, \cdots, \rho(k) .
\end{aligned}
$$

Proof. We write, for some $\theta^{\prime}$ such that $0<\theta^{\prime}<\theta$,

$$
H(z)=\sum_{k} \sum_{s}\left(a_{k s} p^{-\theta^{\prime}(s-1)}\right) z^{s-1} e^{\left(\omega_{k} p^{-\theta^{\prime}}\right)} z
$$

and observe that our data become

$$
\text { ord } H^{(t-1)}\left(p^{\theta^{\prime}} \alpha_{h}\right) \geqq \eta-(t-1) \theta^{\prime}, \quad \text { for all }(h, t) \text {. }
$$

Let $\nu$ be some positive real number to be determined below, and according to Lemma 8 choose a real number $l$ such that $p^{\prime} H(z)$ is a normal function but $p^{1-\nu} H(z)$ is not a normal function. Let

$$
-l^{\prime}=\min _{(h, t)}\left\{\eta-(T-t) \varepsilon-\text { ord }(t-1) !-(T-1) \theta^{\prime}\right\},
$$

so that according to Lemma $4, p^{\prime \prime} P(z)$ is a normal function, $P(z)$ being the interpolation polynomial which coincides with $H(z)$ at the appropriate $T$ points $p^{\theta^{\prime}} \alpha_{1}, \cdots, p^{\theta^{\prime}} \alpha_{n}$, counting them according to multiplicity.

We assert that for each $\lambda=1,2, \cdots, \sigma$

$$
\text { ord } P^{\left(\lambda-l^{\prime}\right.}(0)<\left(T-(\lambda-1) \theta^{\prime}+\operatorname{ord}(\lambda-1) !-\max \left\{l, l^{\prime}\right\},\right.
$$


which is to say, we claim that Lemma 5 implies that

$$
\begin{aligned}
\operatorname{ord} H^{(\lambda-1)}(0) \geqq \operatorname{ord} P^{(\lambda-1)}(0) \geqq & \min _{(h, t)}\{\eta-(T-t) \varepsilon-\operatorname{ord}(t-1) ! \\
& \left.-(\lambda-1) \theta^{\prime}+\operatorname{ord}(\lambda-1) !\right\},
\end{aligned}
$$

the latter bound being obtained by grace of Lemma 1 .

For suppose that, on the contrary, it happens that $l^{\prime} \geqq l$ and

$$
\begin{gathered}
\operatorname{ord} H^{(\lambda-1)}(0) \geqq(T-(\lambda-1)) \theta^{\prime}+\operatorname{ord}(\lambda-1) !-l^{\prime} \\
=\min _{(h, t)}\left\{\eta-(T-t) \varepsilon-\operatorname{ord}(t-1) !-(\lambda-1) \theta^{\prime}-\operatorname{ord}(\lambda-1) !\right\}+\theta^{\prime} .
\end{gathered}
$$

Then, since $\theta^{\prime}>0$, a fortiori our assertion is the case and (6) holds. In the alternative case, suppose that, contrary to the assertion, we have $l>l^{\prime}$ and

$$
\text { ord } H^{(l-1)}(0) \geqq(T-(\lambda-1)) \theta^{\prime}+\text { ord }(\lambda-1) !-l \quad \lambda=1,2, \cdots, \sigma .
$$

It is convenient to write $H^{(\lambda-1)}(0)=H_{\lambda-1}, \lambda=1,2, \cdots$. We now observe that the $H_{\lambda-1}$ satisfy the linear homogeneous recurrence relation

$$
H_{\sigma+\mu}=\beta_{1} H_{\sigma-1+\mu}+\cdots+\beta_{\sigma} H_{\mu}, \quad \mu=0,1,2, \cdots,
$$

where

$$
\prod_{k=1}^{m}\left(X-\omega_{k} p^{-\theta^{\prime}}\right)^{\rho(k)}=X^{\sigma}-\beta_{1} X^{\sigma-1}-\cdots-\beta_{\sigma} .
$$

Because ord $\beta_{\lambda}>\lambda /(p-1), \lambda=1,2, \cdots, \sigma$, we obtain on multiple application of the relations (8), that for $\mu=0,1,2, \cdots$.

$$
\begin{aligned}
\operatorname{ord} H^{(\sigma+\mu)}(0)> & \min _{1 \leqq \lambda \leqq \sigma}\left\{\left(T-(\lambda-1) \theta^{\prime}+\operatorname{ord}(\lambda-1) !\right.\right. \\
& +(\sigma+\mu-\lambda+1) /(p-1)-l\}
\end{aligned}
$$

Moreover, by Lemma 6 it is the case that

$$
(\sigma+\mu) /(p-1) \geqq \operatorname{ord}(\sigma+\mu) !+p^{-\left(\log _{p}(\sigma+\mu)\right.}(\sigma+\mu) /(p-1),
$$

so that (9) becomes for $\mu=0,1,2, \cdots$.

$$
\begin{aligned}
& \text { ord } H^{(\sigma+\mu)}(0)-\operatorname{ord}(\sigma+\mu) ! \\
& \geqq(T-(\sigma-1)) \theta^{\prime}-l+p^{-f \log _{p}(\sigma+\mu) 1}(\sigma+\mu) /(p-1) \\
& \quad+\min _{1 \leqq \lambda \leqq \sigma}\left\{(\sigma-\lambda) \theta^{\prime}+\operatorname{ord}(\lambda-1) !-(\lambda-1) /(p-1)\right\} .
\end{aligned}
$$

A very careful check of the behaviour of the last term on the right hand side shows that, no matter how small $\theta$ might be, the minimum is no smaller than $-\left[\log _{p}(\sigma+p-1)\right]$; more precisely, it is not less than

$$
-\max _{0 \leqq r \leqq p-1}\left\{\left[\log _{p}(\sigma+r)\right]-r /(p-1)\right\} .
$$


Of course if $\theta$ is at all large, say $\theta \geqq 1 /(p-1)$ then without ado the minimum is attained at $\lambda=\sigma$ and in any event is no smaller than $-((\sigma-1) /(p-1)-$ ord $(\sigma-1) !)$.

It follows that if $T \geqq(\sigma-1)+[\log (\sigma+p-1) / \log p] / \theta$ then the inequalities (10) inform us that for $\mu=0,1,2, \cdots$.

$$
\begin{aligned}
\text { ord } H^{(\sigma+\mu)}(0)-\operatorname{ord}(\sigma+\mu) ! \geqq & {\left[\log _{p}(\sigma+p-1)\right]\left(\theta^{\prime} / \theta-1\right)-l } \\
& p^{-\left[\log _{p}(\sigma+\mu)\right]}(\sigma+\mu) /(p-1) .
\end{aligned}
$$

Similarly the inequalities (7) then become, for $\lambda=1,2, \cdots, \sigma$

$$
\text { ord } H^{(\lambda-1)}(0)-\operatorname{ord}(\lambda-1) ! \geqq\left[\log _{p}(\sigma+p-1)\right] \theta^{\prime} / \theta+(\sigma-\lambda) \theta^{\prime}-l
$$

We are left free to suppose that $\theta-\theta^{\prime}>0$ is arbitrarily small and we note that

$$
1 /(p-1) \leqq p^{-\left[\log _{p}(\sigma+\mu)\right]}(\sigma+\mu) /(p-1)<p /(p-1) .
$$

Accordingly there is a real number $\nu$ satisfying $0<\nu<1 /(p-1)$ such that, for $\theta^{\prime}$ sufficiently close to $\theta$, the inequalities (11) and (12) miraculously imply that for $\mu=0,1,2, \cdots$.

$$
\text { ord } H^{(\mu)}(0)-\text { ord } \mu ! \geqq-l+\nu
$$

Since we have chosen $\nu$ independently of $l$ the requirements of Lemma 8 are satisfied and we may conclude that the normality of $p^{l-\nu} H(z)$ constitutes a contradiction. It follows that the inequality (6) holds.

Hence, applying Lemma 7 to $H(z)$ under the condition that $\theta^{\prime}$ is sufficiently close to $\theta$, we obtain for all $(k, s)$

$$
\begin{aligned}
& \text { ord } a_{k s}-(s-1) \theta^{\prime} \\
& >\min _{\lambda}\left\{\operatorname { m i n } _ { ( h , t ) } \left\{\eta-(T-1) \varepsilon-(\sigma-s) \delta_{k}-\operatorname{ord}(s-1) !\right.\right. \\
& +(\sigma-1) /(p-1)+(\sigma-\lambda) \theta^{\prime}+\operatorname{ord}(\lambda-1) !-(\lambda-1) /(p-1)-(s-1) \theta^{\prime} \\
& +(t-1) \varepsilon-\operatorname{ord}(t-1) !\}\} \text {. }
\end{aligned}
$$

The minimum over $\lambda$ has already been studied above, whence we promptly obtain the assertion of the theorem.

Corollary 1. With the notation of the theorem, suppose $\sigma>1$. Then already if $T \geqq(\sigma-1)+\max _{0 \leqq r \leqq p-1}\{[\log (\sigma+r) / \log p]-r /(p-1)\} / \theta$ we obtain that for all $(k, s)$,

$$
\begin{aligned}
\text { ord } a_{k s}> & \eta-(T-1) \varepsilon-(\sigma-s) \delta_{k}-\operatorname{ord}(s-1) ! \\
& -\max _{0 \leq r \leq p-1}\{[\log (\sigma+r) / \log p]-r /(p-1)\}+(\sigma-1) /(p-1) \\
& +\min _{(n, t)}\{(t-1) \varepsilon-\operatorname{ord}(t-1) !\}
\end{aligned}
$$


If moreover $\varepsilon \geqq 1 /(p-1)$, we obtain for all $(k, s)$,

$$
\text { ord } \begin{aligned}
a_{k s}> & \eta-(T-1) \varepsilon-(\sigma-1) \delta_{k}-\max _{0 \leqq r \leqq p-1}\{[\log (\sigma+r) / \log p] \\
& -r /(p-1)\}+(\sigma-1) /(p-1) \geqq \eta-(T-1) \varepsilon-(\sigma-1) \delta_{k} .
\end{aligned}
$$

Proof. The above statements are respectively the best possible, and the optimally simple statements of the theorem. We could include the (trivial) case $\sigma=1$ by requiring that $T$ be strictly greater than the above bound, that is, that $T \geqq 1$. The details which lead to the best possible statement are already included in the proof of the theorem.

Theorem 3. Let $F$ be a p-adic exponential polynomial, the distinct frequencies $\omega_{k}$ of which satisfy ord $\omega_{k} \geqq 1 /(p-1)+\theta$, for some $\theta>0, k=$ $1, \cdots, m$. Suppose that the degree of $F$ is $\sigma>1$. Then the number of zeros of $F$ in the $\operatorname{disc}\left\{z:|z|_{p} \leqq 1\right\} \subset \Omega_{p}$ is less than

$$
(\sigma-1)+\max _{0 \leq r<p-1}\{[\log (\sigma+r) / \log p]-r /(p-1)\} / \theta,
$$

or $F$ vanishes identically.

Proof. In the corollary above suppose that $F$ has as many as $T$ zeros, so that $\eta=\infty$. Then it follows that for all $(k, s)$, ord $a_{k s}=\infty$ which is to say that $F$ vanishes identically.

Remarks. (i) It would seem that Theorem 3 is exact. Indeed in van der Poorten (197?) I showed by a slightly different method (Strassman's theorem, or, equivalently, the $p$-adic Weierstrass preparation theorem) that the number of zeros of $F$ in $|z|_{p} \leqq 1$ does not exceed $(\sigma-1)(1+1 /(p-1) \theta)$. This essentially coincides with Theorem 3 when $\sigma<p$. The method of van der Poorten (197?) is potentially exact and, with great care, one could no doubt use it to obtain Theorem 3.

(ii) It is of great interest to observe that in Theorem 3 the number of zeros of $F$ may exceed $\sigma-1$ by an amount which depends, albeit only logarithmically, on $\sigma$. One easily writes down the analogue of the complex local valency results (see Tijdeman (1971) and W. K. Hayman (1973).

(iii) Our results when, say compared to those of Shorey (1972a, b) obtained by a $p$-adic paraphrase of the complex method, suggest that there is considerable advantage to be obtained by avoiding the Schnirelman integral and using, in place thereof, a suitable interpolation formula. We hope to vindicate this viewpoint more forcefully in a future paper on a $p$-adic version of Baker's theorem on linear forms in the logarithms of algebraic numbers.

(iv) Recent work of Baker, see also remarks in Cijsouw and Tijdeman (1975), suggests that the natural way of viewing an exponential polynomial has it of the shape 


$$
F(z)=\sum_{k=1}^{m} \sum_{s=1}^{\rho(k)} \frac{b_{k s}}{(s-1) !} z^{s-1} e^{\omega_{k} z},
$$

whilst the data are bounds for $\left(F^{(t-1)}\left(\alpha_{h}\right)\right) /(t-1)$ ! (rather than for $F^{(t-1)}\left(\alpha_{h}\right)$ ). Our result confirms this view, and we quote the appropriate conclusion as a further corollary of Theorem 2.

COROLLARY 2. With the notation of the theorem, suppose $\sigma>1$ and write for each $(k, s), b_{k s}=a_{k s}(s-1)$ !. Then

$$
T \geqq(\sigma-1)+\max _{0 \leqq r<p-1}\{[\log (\sigma+r) / \log p]-r /(p-1)\} / \theta
$$

and

$$
\operatorname{ord}\left(F^{(t-1)}\left(\alpha_{h}\right) /(t-1) !\right) \geqq \chi, \quad \text { for } h=1, \cdots, n ; t=1, \cdots, \tau(h)
$$

together imply that for each $(k, s)$

$$
\begin{aligned}
\text { ord } b_{k s}> & \chi-(T-1) \varepsilon-(\sigma-s) \delta_{k}+((\sigma-1) /(p-1) \\
& \left.-\max _{0 \leq r<p-1}\left\{\left[\log _{p}(\sigma+r)\right]-r /(p-1)\right\}\right) .
\end{aligned}
$$

Proof. This is just (13) in Corollary 1.

\section{Details in particular cases}

As already remarked, it is quite easy to recover any detail which, in a particular case might provide a stronger result than is available from Lemma 1 and Theorem 2 and its corollaries. We have in mind applications to the auxiliary functions that appear in Baker's work (see, for example Baker (1971)) and accordingly will concentrate on the case already alluded to in Lemma 2 . Incidentally, that result, together with Lemma 5, replaces the extrapolation lemma in $p$-adic versions of Baker's work (see, for example, Baker and Coates (1975)). It is easily seen that additional detail concerning the interpolation points $\alpha_{1}, \cdots, \alpha_{n}$ will not disturb our results other than permitting us to replace the term $(T-1) \varepsilon$ by some smaller quantity; however in (10) it may force us to choose the minimum over $\lambda$ at $\lambda=\sigma$ providing a minor additional sharpening of the inequalities.

Lemma 9. Let $\operatorname{ord}_{k \neq h}\left(\alpha_{h}-\alpha_{k}\right)=\varepsilon_{h k}$ and $\max _{k \neq h} \varepsilon_{h k}=\varepsilon_{h}, h, k=1, \cdots, n$. Similarly let $\operatorname{ord}_{h \neq k}\left(\omega_{k}-\omega_{h}\right)=\delta_{k h}$ and $\max _{h \neq k} \delta_{k h}=\delta_{k}, k, h=1, \cdots, m$. Then in Corollary 2 of Theorem 2 we may replace the quantity $(T-1) \varepsilon$ by $\max _{h}\left\{\Sigma_{k \neq h} \tau(k) \varepsilon_{h k}+(\tau(h)-1) \varepsilon_{h}\right\}$, and the quantity $(\sigma-s) \delta_{k}$ by $\Sigma_{h \neq k} \rho(h) \delta_{k h}+$ $(\rho(k)-s) \delta_{k}$. 
ProOF. Simply make the indicated replacements throughout, checking that they verify Lemma 1 and Lemma 7.

The following result is a $p$-adic analogue of Theorem 1 of Cijsouw and Tijdeman (1975)

THEOREM 4. Let F be a p-adic exponential polynomial

$$
F(z)=\sum_{k=1}^{m} \sum_{s=1}^{\rho(k)} \frac{b_{k s}}{(s-1) !} z^{s-1} e^{\omega_{k^{2}}},
$$

with coefficients $b_{k s}$, not all zero, in $\Omega_{p}$, and distinct $f$ equencies $\omega_{k}$ in $\Omega_{p}$ satisfying ord $\omega_{k} \geqq 1 /(p-1)+\theta$, some $\theta>0$ and the crnditions of Lemma 9 . Let $\sigma=\Sigma \rho(k)>1$ be the degree of $F$.

Further let $R S=T$ where $R, S$ are positive integers. Then

$$
T \geqq(\sigma-1)+\max _{0 \leqq r<p-1}\{[\log (\sigma+r) / \log p]-r /(p-1)\} / \theta
$$

and

$$
\operatorname{ord}\left\{F^{(t-1)}(h) /(t-1) !\right\} \geqq \chi \quad \text { for } h=1, \cdots, R ; t=1, \cdots, S
$$

imply that

$$
\begin{aligned}
\text { ord } b_{k s}> & \chi-(S \operatorname{ord}(R-1) !+(S-1)[\log (R-1) / \log p]) \\
& -\left(\sum_{h \neq k} \rho(h) \delta_{k h}+(\rho(k)-s) \delta_{k}\right) \\
& +\left((\sigma-1) /(p-1)-\max _{0 \leqq r<p-1}\{[\log (\sigma+r) / \log p]-r /(p-1)\}\right)
\end{aligned}
$$

for all $k=1, \cdots, m ; s=1, \cdots, \rho(k)$.

Proof. This is Corollary 2 of Theorem 2 together with the detail indicated in Lemma 9, and the observation that

$$
\begin{gathered}
\max _{1 \leq h \leqq R}\left\{S \operatorname{ord}(R-h) !(h-1) !+(s-1) \max _{1 \leq j \neq h \leq R} \operatorname{ord}(h-j)\right\} \\
=S \operatorname{ord}(R-1) !+(S-1)[\log (R-1) / \log p]
\end{gathered}
$$

REMARK. There may be purpose in replacing the polynomial coefficients in the exponential polynomial, so that $\sum_{s} a_{k s} z^{s-1}$ becomes $\sum_{s} c_{k s}(z+1)$ $z+2) \cdots(z+S) /(S-1) !, k=1, \cdots, m$. If so, it is easy to show that (see Cijsouw and Tijdeman (1975), Lemma 4)

$$
\text { ord } c_{k s} \geqq \min _{1 \leqq t \leqq \rho(k)} \text { ord } a_{k t}=\min _{1 \leqq t \leqq \rho(k)}\left\{\text { ord } b_{k t}-\text { ord }(t-1) !\right\} .
$$




\section{About applications}

There are good examples of applications of the complex analogues of Theorem 3 and respectively Theorem 4 to transcendence arguments, in Waldschmidt (1974), Chapter 7 and respectively Cijsouw (1975). In Shorey (1972a) and (1972b) there are similar applications of earlier cruder forms of Theorem 3, and respectively Theorem 2 .

One can replace the $p$-adic extrapolation lemma (an integral form of the Hermite interpolation formula) by our exact formula, Theorem 1 (which is just the Hermite interpolation formula) and, as examples, Lemma 1 and Lemma 2, and then apply Lemma 5.

There is an interesting conjecture to the effect that if a recurrence sequence of integers has only finitely many zeros then this number of zeros is bounded by a constant dependent only on the order of the recurrence. There is reason to believe that this question can be approached with the aid of the very precise results of Theorem 2 and Theorem 3.

\section{References}

A. Baker (1971), 'Recent advances in transcendence theory', Proc. International Conference on Number Theory. Moscow, September, 10, 67-69.

A. Baker and J. Coates (1975), 'Fractional parts of powers of rationals', Proc. Camb. Phil. Soc. 77 , 269-279.

P. L. Cijsouw (1975), 'On the simultaneous approximation of certain numbers', Duke Math. J., 42, 249-257.

P. L. Cijsouw and R. Tijdeman (1975), 'An auxiliary result in the theory of transcendental numbers II', Duke Math. J. 42, 239-247.

W. K. Hayman, 'Differential inequalities and local valency', Pacific J. Math. 44, 117-137.

K. Mahler (1932), 'Zur Approximation der Exponentialfunktion und des logarithmus', J. reine und angew. Math., 166, 118-150.

K. Mahler (1935), 'Über transzendente P-adische Zahlen', Comp. Math. 2, 259-275.

K. Mahler (1967a), 'On a class of entire functions', Acta. Math. Acad. Sci. Hungar. 18, 83-96.

K. Mahler (1967b), 'Applications of some formulae by Hermite to the approximation of exponentials and logarithms', Math. Annalen 168, 200-227.

A. Schinzel (1967), 'Two theorems of Gelfond and some of their applications', Acta Arith. 13, 177-236.

T. N. Shorey (1972a), 'Algebraic independence of certain numbers in the p-adic domain', Indag. Math. 34, 423-435.

T. N. Shorey (1972b), ' $P$-adic analogue of a theorem of Tijdeman and its application', Indag. Math. 34, 436-442.

R. Tijdeman (1971), 'On the number of zeros of general exponential polynomials', Indag. Math. 33, $1-7$.

R. Tijdeman (1973), 'An auxiliary result in the theory of transcendental numbers', $J$. Numb. Theory $5,80-94$.

A. J. van der Poorten (1970), 'Generalisations of Turán's main theorems on lower bounds for sums of powers', Bull. Austral. Math. Soc. 2, 15-38. 
A. J. van der Poorten (1975), 'Some determinants that should be better known', J. Austral. Math. Soc. 21, 278-288.

A. J. van der Poorten (197?), 'Zeros of $p$-adic exponential polynomials', Indag. Math. (to appear). M. Waldschmidt (1974), Nombres Transcendants, Springer Lecture Notes 402.

School of Mathematics

The University of New South Wales

Australia 